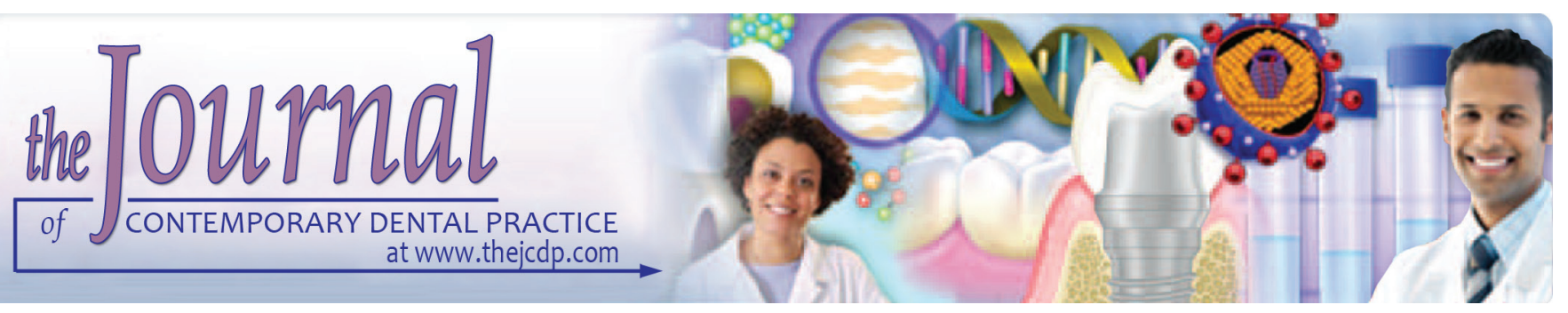

\title{
Assessment of Antibacterial and Antifungal Properties and In Vivo Cytotoxicity of Peruvian Passiflora mollisima
}

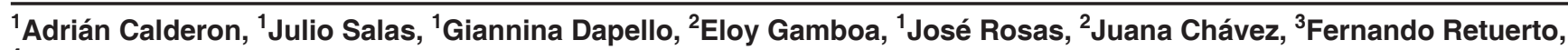
${ }^{4}$ Frank Mayta-Tovalino

\begin{abstract}
Aim: The antimicrobial activity of Passiflora mollissima is largely due to its aliphatic and aromatic hydrocarbons, terpenes, alkaloids, ketones, esters, alcohols, and lactones. The objective of this research was to evaluate the antimicrobial activity of the compounds in the Passiflora mollissima (Tumbo) fruit and leaves included also on cultured strains of the microorganisms Streptococcus mutans, Streptococcus oralis, Streptococcus sanguinis and Candida albicans.
\end{abstract}

Materials and methods: Antimicrobial activity was analyzed by the disk diffusion method and evaluated in terms of their zones of inhibition. The cytotoxic activity of these compounds was determined in a bioassay involving sea urchin eggs (Tetrapygusniger) in which the inhibition percentage of artificially fertilized ovules was equivalent to their cytotoxic activity after 26 hours.

Results: This study demonstrated the antimicrobial activity of ethanolicPassiflora mollissima extract against the cultured strains of Streptococcus mutans, Streptococcus oralis, and Streptococcus sanguinis with zones of inhibition after the incubation period.

Conclusion: There was no evidence of activity against the pathogenic Candida albicans, which demonstrates a null antifungal capacity of the evaluated extracts. However, cytotoxic activity was found, and the compounds led to the complete inhibition of $100 \%$ of sea urchin ovules after 26 hours of exposure.

${ }^{1}$ School of Stomatology, Department of Health Sciences, Universidad Privada San Juan Bautista, Lima, Republic of Peru

${ }^{2}$ Department of Microbiology, Faculty of Health Sciences, Universidad Privada San Juan Bautista, Lima, Republic of Peru

${ }^{3}$ Faculty of Biological Sciences, Department of Cellular Biology and Genetics, Universidad Nacional Mayor de San Marcos, Lima, Republic of Peru

${ }^{4}$ Faculty of Health Sciences, Department of Public Health, Universidad Privada San Juan Bautista, Lima, Republic of Peru

Corresponding Author: Frank Mayta-Tovalino, Faculty of Health Sciences, Universidad Privada San Juan Bautista, Lima, Republic of Peru, e-mail: estadistico2.0@gmail.com
Clinical importance: The results of the study serve as a guide. in the industrial use of this natural resource "tumbo" due to the discovery of a potential medicine based on this plant that would be a great development in the field of antimicrobial therapies.

Keywords: Antibacterial activity, Antifungal activity, Cytotoxicity, Passiflora mollisima, Zone of inhibition.

How to cite this article: Calderon A, Salas J, Dapello G, Gamboa E, Rosas J, Chávez J, Retuerto F, Mayta-Tovalino F. Assessment of Antibacterial and Antifungal Properties and In Vivo Cytotoxicity of Peruvian Passiflora Mollisima. J Contemp Dent Pract 2019;20(2):145-151.

Source of support: Nil

Conflict of interest: None

\section{INTRODUCTION}

The biodiversity of natural flora currently provides multiple applications in different areas of research. According to several authors, ${ }^{1-7}$ there are approximately 3000 species of plants that contain essential oils, of which 300 are in commercial use, and their compounds are used in different fields of industry. ${ }^{8,9}$ During the last decade, microorganisms have developed greater antibiotic resistance, and thus, there has been an increase in the development of new antibiotics in search of solutions to this problem. Knowing the uncertain future for the existing antimicrobial drugs and bearing in mind that in ancient times plants were invaluable sources for supporting human health, plants have become important resources for obtaining drugs over the past decade, according to the World Health Organization. ${ }^{10-12}$ Antibacterial resistance is a serious health problem, and thus, it is important to find agents with new modes of action. The plants used in medicinal therapy present components that show physiological and pharmacological activities, which are effective antimicrobials for treating infectious diseases and can simultaneously mitigate the adverse effects associated with these illnesses. ${ }^{13-16}$ 
Passiflora mollissima is a climbing plant native to the inter-Andean valleys of South America from 1,000 to 3,500 m.a.s.l. ${ }^{2}$ It grows between the valleys of Ancash, Junín, Moquegua, Huancavelica, and Abancay in Perú, and it is used as food, as medicine, and for ornamental purposes. ${ }^{2,17}$ There is a wide variety of Passiflora species that have been used in many countries as traditional medicines. ${ }^{2-7}$ The fruits of this Passiflora plant contain various components such as alkaloids, phenols, glycosyls, flavonoids, and cyanogenic compounds. ${ }^{3,6,17-19}$ For example, since 1973, Birner and Nicolls have shown the antimicrobial activity of Passiflora mollissima through its active agent, passicol. Studies have revealed sedative, antispasmodic and anxiolytic activities, which are qualities that have not been attributed to this active component until now. ${ }^{20}$

The purpose of this investigation was to compare the in vitro antimicrobial effect of an ethanolic Passiflora mollissima extracts on cultured strains of Streptococcus mutans, Streptococcus oralis, Streptococcus sanguinis and Candida albicans and to demonstrate the cytotoxic activity of this natural resource in vivo because they are some of the most representative microorganisms of the oral microflora, besides these microorganisms are part of the normal microflora of the oral cavity of humans. ${ }^{21-25}$

\section{MATERIALS AND METHODS}

The present in vitro study was carried out in the Multifunctional Science Laboratory of the Faculty of Health Sciences, San Juan Bautista Private University, Lima, Peru.

\section{Fruit Collection and Extract Preparation}

Ten kilograms of a natural raw sample of Passiflora mollissima fruit, which in Peru is known as "tumbo", was collected from the mountain region of Peru in the Pachachaca valley at an approximate altitude of 2150 meters above sea level. The fruits were washed with distilled water and dried, and its leaves and stems were removed to eliminate any polluting agent. The study of the plant was carried out by the San Marcos Herbarium of the Natural History Museum of the Universidad Nacional Mayor de San Marcos with code N 098-USM-2017, having the following classification:

- Division: Magnoliophyta

- Subclass: Magnoliopsida

- Order: Violaes

- Family: Passifloraceae

- Gender: Passiflora

- Species: Passiflora tripartita var. mollisima (Kunth) Holm-Niels and P Jorg.

The pulp was submitted to a high-pressure extraction to obtain a liquid product. As a result of this procedure,
$3200 \mathrm{~mL}$ of pure extract from the Passiflora mollissima juice was obtained, to which the solvent medium was added later. In this case, the solvent consisted of $1000 \mathrm{~mL}$ of ethyl alcohol at $70^{\circ}$. The new mixture was stored at room temperature in an air-tight maceration bottle in the dark. ${ }^{5}$ After that, the alcoholic preparation was allowed to rest for 10 days, with stirring three times per day before the filtering process using cotton for coarse substrates and filter paper for fine substrates. The result was a $3950 \mathrm{~mL}$ solution of concentrated extract, which was transferred to a $40 \times 27 \mathrm{~cm}$ Pyrex container to evaporate the ethanol in a "Memmert" model UE 500 laboratory oven for 8 days at a constant temperature of $45^{\circ} \mathrm{C}$ to obtain a pure extract. Subsequently, the pure ethanol extract was diluted to different concentrations $(25,50,75 \%)$ in a distilled water medium, to perform the antimicrobial test. ${ }^{5}$

\section{Reactivation of Microbial Strains}

The microbiological cultured method was blood agar for gram-positive microorganisms of the streptococcaceae family. ${ }^{21,26}$ This study used Streptococcus mutans (ATCC 25175), Streptococcus oralis (ATCC 6249), and Streptococcus sanguinis (ATCC 10556). The extract solution was transferred to $150 \times 30 \mathrm{~mm}$ Petri plates containing TSA agar and nutrient agar as the culture medium. Using the lawn plate method procedure, the samples were labeled, plated and placed in an Auto-Flow UN-4950 laboratory incubator at a temperature of $37^{\circ} \mathrm{C}$ for 24 hours, after which a positive growth of the bacteria was obtained from their culture media. To standardize the assay, the McFarland scale was used to estimate $10^{8} \mathrm{UFC}$ qualitatively for all the cultures grown here. Finally, the plate containing previously cultured blood agar was incubated in the same incubator for 24 hours at a constant temperature of $37^{\circ} \mathrm{C} .^{2}$ To cultivate the Candida albicans, sabouraud dextrose agar was used as a culture medium, then placed in an Auto-Flow UN-4950 laboratory incubator for 24 hours at a constant temperature of $37^{\circ} \mathrm{C} .{ }^{27}$

\section{In vitro antimicrobial susceptibility testing of Passiflora mollissima}

The bacterial and fungal strains were obtained from the GenLab del Perú SAC. The company, which provided bio-tubes containing the lyophilized strains and their respective certificates of analysis to guarantee the culture. The bacterial strains were cultured at $37^{\circ} \mathrm{C}$ for 24 hours on blood agar, while the fungal strain was cultured for 48 hours at $37^{\circ} \mathrm{C}$ on Sabouraud dextrose agar. ${ }^{5}$

The antimicrobial test was performed by the diffusion disc method; $100 \mu \mathrm{L}$ suspensions containing approximately $10^{8}$ colony-forming units (CFU)/mL of bacterial cells and $10^{4}$ cells $/ \mathrm{mL}$ of fungal cells were spread 
on blood agar and Sabouraud dextrose agar, respectively. Filter paper discs were impregnated with $100 \mu \mathrm{L}$ of the fruit extract concentrations (25, 50, 75 y 100\%) and placed into the inoculated agar plates.

All the Petri dishes were plated and labeled, and then they were incubated at a temperature of $37^{\circ} \mathrm{C}$ for 24 hours. The antimicrobial activity was subsequently evaluated by measuring the diameter of the zone of inhibition, in which it was observed that the growth of the bacterial colonies had disappeared. Finally, the diameter of the inhibition zones against the positive control (ampicillin and miconazole) groups were contrasted respectively. ${ }^{2}$

\section{Cytotoxic Quantification of Passiflora mollissima in Sea Urchin Eggs (Tetrapygus niger)}

The evaluation of alterations in the development of sea urchin eggs (Tetrapygus niger) has been considered as a reliable and adequate method for cytotoxic evaluation. For that reason, to evaluate genotoxic effects, embryotoxicity and teratogenicity artificially fertilized embryos of sea urchins were used. The initial results suggest that this bioassay has the advantage to be a sensitive indicator for cytotoxic and mutagenic contaminants; this type of assay is characterized by its rapidity, low cost, and applicability to a variety of toxicants. ${ }^{28}$

The bioassay chosen to evaluate the cytotoxic effect of the ethanolic extract from the Passiflora mollissima fruit was the lethality of Tetrapygus niger eggs. This assay involved the collection of 10 sea urchins at 6 am due to the lower stress and higher possibility of finding sperm at that hour. After the sample was transported to a glass container in $3 \mathrm{~L}$ of prefiltered seawater, it was dispensed into nine clear sterile $10 \mathrm{~mL}$ bottles with their respective labels and divided into three equivalent concentration groups to assess the concentrations of $0.125 \mu \mathrm{L} / \mathrm{mL}, 0.25$ $\mu \mathrm{L} / \mathrm{mL}$, and $0.375 \mu \mathrm{L} / \mathrm{mL}$. One male sea urchin was selected for its sperm, and a female sea urchin was used for its ovules; the gonads were placed into a glass sterile beaker containing $150 \mathrm{~mL}$ of filtered seawater for the artificial insemination of the sea urchin eggs. The flask in which fertilization took place then became the control group to which no agent was added. ${ }^{29}$

The vial was left in a Zhicheng SZD-1090 incubator at a constant temperature of $15.3^{\circ} \mathrm{C}$. BELNET microscope confirmed the fertilization of the sea urchin eggs, the procedure was continued by adding $1 \mathrm{~mL}$ of filtered seawater and $3 \mathrm{~mL}$ of seawater containing the fertilized ovules to each of the nine flasks. Finally, to each of the groups of 3 bottles, the ethanolic Passiflora mollissima extract was added at different concentrations (first group: $0.5 \mu \mathrm{L}$, second group: $1.0 \mu \mathrm{L}$ and a third group: $1.5 \mu \mathrm{L}$ ) to obtain the $0.125 \mu \mathrm{L} / \mathrm{mL}, 0.25 \mu \mathrm{L} / \mathrm{mL}$ and $0.375 \mu \mathrm{L} / \mathrm{mL}$ concentrations, respectively. ${ }^{29}$

\section{Statistical Analysis}

For the univariate analysis, we measured the mean, standard deviation for the variable antimicrobial effects of the ethanolic extract. Its potential cytotoxic effect was determined in the same way. Each sample was tested for normal distribution using the Shapiro-Wilk test. For the bivariate analysis, ANOVA was used to compare the antimicrobial effect of the extract against the different strains of cultured microorganisms, and the same was performed to gauge its cytotoxic effect. The results were considered significant when the $p$ value of $<0.05$.

The database was prepared using the Microsoft Excel program, and the results were analyzed using the statistical package Stata ${ }^{\circledR}$ version 12.0.

\section{Ethics Statement}

This study included sea urchin (Tetrapygus niger) biomodels, and it was performed in accordance with international animal welfare guidelines. The protocols were approved by the Institutional Ethics Committee of the Universidad Privada San Juan Bautista under code CEPB-FCS0007. All the animals used in this study were conventionally housed in the facilities of the Basic Sciences Laboratory.

\section{RESULTS}

\section{In Vitro Antimicrobial Activity}

According to the in vitro analysis on the antimicrobial effects of the ethanolic extract of the Passiflora mollissima fruit at four concentrations (25\%,50\%, 75\% and 100\%) in four cultured strains of microorganisms found in the oral flora (S. mutans, S. oralis, S. sanguinis and C. albicans), the $100 \%$ ethanolic fruit extract created the largest inhibition halos for the $S$. mutans strain, with a mean of $12.8 \pm 2.0 \mathrm{~mm}$, followed by a mean of $8.8 \pm 0.9 \mathrm{~mm}$ for the concentration at $75 \%$, a mean of $7.4 \pm 0.9 \mathrm{~mm}$ for the $50 \%$ concentration and no antimicrobial effect for the $25 \%$ concentration. Likewise, the $100 \%$ ethanol fruit extract achieved the largest inhibition halos against the $S$. oralis strain, with a mean of $15.4 \pm 2.0 \mathrm{~mm}$, followed by a mean of $11.7 \pm 2.0 \mathrm{~mm}$ concentration for $75 \%$, a mean of $9.0 \pm$ $1.9 \mathrm{~mm}$ for the $50 \%$ concentration and no antimicrobial activity for the $25 \%$ concentration. However, the ethanolic fruit extract did not present any antimicrobial effect at any of its four concentrations against the C. albicans strain (Table 1).

When a statistical inference was made between the means of the concentrations of the ethanolic fruit extract and the cultured strains evaluated here, a statistically significant difference was found for the $75 \%$ concentration $(p=0.017)$. On the other hand, there was no statistically 
significant difference for the $50 \%$ concentration ( $p=$ 0.203 ) or for the $100 \%$ concentration $(p=0.063)$ on the cultured strains. Furthermore, at the 25\% concentration, no statistically significant difference could be found, as reflected by the null results (Table 2).

\section{Cytotoxic Activity}

Finally, the cytotoxicity was evaluated by an in vitro bioassay of sea urchin eggs (Tetrapygusniger). The results suggest that the ethanolic fruit extract of Passiflora mollissima presents mild cytotoxicity at $0.125 \mu \mathrm{L} / \mathrm{mL}$, with a mean lethality of $25.6 \%$ during the first 6 hours; however, increasing the concentration to $0.25 \mu \mathrm{L} / \mathrm{mL}$ and $0.375 \mu \mathrm{L} / \mathrm{mL}$ ethanolic fruit extract causes high cytotoxicity in sea urchin embryos which evolutionary stage was at 6 hours, with means of $90.6 \%$ and $92.3 \%$, respectively. Embryonic cleavage upon 26 hours of exposure indicated that all three concentrations of the ethanolic fruit extract had a high cytotoxic effect on the sea urchin embryos, causing total mortality, with a mean of $100 \%$ for all cases (Table 3 ).

\section{DISCUSSION}

The promising antibacterial and medicinal effects of fruits found in nature continue to highlight the natural world as a source of plant-based drugs containing herbal extracts that can be used for different chemotherapeutic purposes. In addition to using the components of natural plants and fruits for the alternative preparation of medicines, this continues to play a greater role in assisting people throughout the world. ${ }^{18}$

The antimicrobial effect was evaluated by means of an antibiogram like procedure, in which the bacterial inhibition diameters were measured in millimeters with a digital caliper. In a similar manner, the data obtained here were used to determine the minimum inhibitory concentration, and the methodology described here was also used by multiple authors. ${ }^{2-7}$ Notably, the cytotoxic effect was determined from an in vitro bioassay on sea urchin embryos of the sixth hour of life (Tetrapygus niger), in which the fertilization of sea urchin ovules was confirmed on a saline medium was artificially simulated and the eggs were subsequently directly exposed to

Table 1: In vitro evaluation of the antimicrobial activity of the ethanolic extract from the Passiflora mollissima fruit at 4 different concentrations against each individual microbial strain

\begin{tabular}{|c|c|c|c|c|c|c|c|c|}
\hline \multicolumn{9}{|c|}{ Concentration of the ethanolic extract of Passiflora mollissima } \\
\hline Cultured strain & $25 \%$ & $p^{*}$ & $50 \%$ & $p^{*}$ & $75 \%$ & $p^{*}$ & $100 \%$ & $p^{*}$ \\
\hline S. mutans & $0 \pm 0$ & - & $7.4 \pm 0.9$ & 0.346 & $8.8 \pm 0.9$ & 0.036 & $12.8 \pm 2.0$ & 0.506 \\
\hline S. oralis & $0 \pm 0$ & - & $9.0 \pm 1.9$ & 0.749 & $11.7 \pm 2.0$ & 0.834 & $15.4 \pm 2.0$ & 0.777 \\
\hline S. sanguinis & $0 \pm 0$ & - & $8.4 \pm 1.3$ & 0.078 & $11.7 \pm 1.1$ & 0.507 & $17.4 \pm 0.4$ & 0.456 \\
\hline Candida albicans & $0 \pm 0$ & - & $0 \pm 0$ & - & $0 \pm 0$ & - & $0 \pm 0$ & - \\
\hline
\end{tabular}

All values are recorded in $\mathrm{mm}$. The concentrations were calculated from the dilutions of the active ingredient

*Shapiro-Wilk test significance level $p<0.05$

Table 2: In vitro comparison of the antimicrobial effect of the ethanolic Passiflora mollissima extract at different concentrations individually, compared to 4 different types of microorganism strains

\begin{tabular}{lcccc}
\hline \multicolumn{5}{c}{ Concentration of the ethanolic extract of Passiflora mollissima } \\
\hline Cultured strain & $25 \%$ & $50 \%$ & $75 \%$ & $100 \%$ \\
\hline S. mutans & $0 \pm 0$ & $7.4 \pm 0.9$ & $8.8 \pm 0.9$ & $12.8 \pm 2.0$ \\
S. oralis & $0 \pm 0$ & $9.0 \pm 1.9$ & $11.7 \pm 2.0$ & $15.4 \pm 2.0$ \\
S. sanguinis & $0 \pm 0$ & $8.4 \pm 1.3$ & $11.6 \pm 1.1$ & $17.4 \pm 0.4$ \\
Candida albicans & $0 \pm 0$ & $0 \pm 0$ & $0 \pm 0$ & $0 \pm 0$ \\
$p^{*}$ & - & 0.203 & 0.017 & 0.000 \\
\hline
\end{tabular}

All values are recorded in $\mathrm{mm}$. The concentrations were calculated from the dilutions of the active ingredient ${ }^{*}$ ANOVA Test

Significance level $p<0.05$

Table 3: In vitro evaluation of the cytotoxic effect of the ethanolic Passiflora mollissima extract on the sea urchin egg (Tetrapygus niger) bioassay

\begin{tabular}{|c|c|c|c|c|c|c|c|c|}
\hline \multirow{2}{*}{$\begin{array}{l}\text { Concentration of the } \\
\text { extract }(\mu L / m L)\end{array}$} & \multicolumn{4}{|c|}{6 hours } & \multicolumn{4}{|c|}{26 hours } \\
\hline & $A(\%)$ & $B(\%)$ & $C(\%)$ & Mean (\%) & $A(\%)$ & $B(\%)$ & $C(\%)$ & Mean (\%) \\
\hline 0.125 & 25 & 27 & 25 & 25.6 & 100 & 100 & 100 & 100 \\
\hline 0.25 & 97 & 92 & 83 & 90.6 & 100 & 100 & 100 & 100 \\
\hline 0.375 & 96 & 91 & 90 & 92.3 & 100 & 100 & 100 & 100 \\
\hline Control & 0 & 1 & 1 & 0.6 & 2 & 2 & 3 & 2.3 \\
\hline
\end{tabular}


the extract. Controlled exposures were subsequently performed over different periods of time to determine the cytotoxic effects, which are shown as the inhibition of fertilized ovule development on a percentage scale. This procedure was used according to the methodology described by other authors. ${ }^{28-31}$

Passiflora mollissima is an exotic fruit native to South America. According to Garcia-Ruiz et al. A total of 18 flavonoid compounds, nine proanthocyanidins, and nine flavan-3-ol monomers were identified and quantified. The glycosides of (epi)-afzelechin which stood out as the most abundant flavonoids. In addition, in their study, they identified three carotenoids, with -carotene having the highest concentration. ${ }^{32}$

Some previous studies performed on the active component passicol, from the Passiflora family, reported that antimicrobial activity can be preserved for a long time as long as the raw material is stored together with an organic solvent in a dark environment, preferably at a temperature of $6^{\circ} \mathrm{C}$. $^{7}$ As part of the technical methodology chosen for the preparation of the Passiflora mollissima fruit extract, ethanol was chosen as a diluent component for the initial crude extract. However, other researchers with similar objectives to those in the present study used diethyl ether, acetone, and ethanol as the diluent components, finding similar antibacterial effects. ${ }^{2,3,5,19}$

The chosen guideline to be able to evaluate an antimicrobial effect using the in vitro assay by the inhibition halo method was based on multiple investigations, namely Kannan et al. in 2010, ${ }^{2}$ Latha et al. in $2015,{ }^{3}$ Ingale et al. in 2010, ${ }^{4}$ Mohanasundari et al. in 2007, Razia et al. in 2014, ${ }^{6}$ and Shiamala et al. in 2014. ${ }^{7}$ The scientific basis used to estimate the cytotoxicity of the ethanolicPassiflora mollissima extract was provided through the sea urchin egg bioassay (Tetrapygus niger). This type of test has previously been used by different authors who found results that support its viability, namely Hose in $1985,{ }^{28}$ Figuerola et al. in $2013,{ }^{30}$ Lin et al. in 2001, ${ }^{31}$ Costa et al. in 2002, ${ }^{33}$ and Gui et al. in 2018, ${ }^{34}$

The results obtained from the present study presume that the ethanolic Passiflora mollissima extract has an antibacterial effect against the strains of bacteria studied here, which were most susceptible at the highest sample concentration of $100 \%$, in the following order, Streptococcus sanguinis (ATCC 10556) $(17.4 \pm 0.4)$, Streptococcus oralis (ATCC 6249) (15.4 \pm 2.0$)$ and lastly Streptococcus mutans (ATCC 25175) $(12.8 \pm 2.0)$. The inhibitory minimum concentration for all these strains is $50 \%(2000 \mu \mathrm{L} / \mathrm{mL})$. However, none of them presented antifungal effects when tested against the fungus Candida albicans (ATCC 10231).

The antimicrobial effect of the ethanolicPassiflora mollissima extract found in the present investigation is consistent with the results published by Kannan et al. ${ }^{2}$ who concluded that a methanolic extract of Passiflora mollissima leaves had a significant antimicrobial effect against the Gram-positive bacteria Staphylococcus aureus and the gram-negative bacteria Salmonella typhi. Additionally, the results are consistent with the conclusions postulated by Mohanasundari C. ${ }^{5}$ in which the leaves and fruits of Passiflora foetida L. had antimicrobial effects against the following human pathogenic bacteria: Pseudomonas putida, Vibrio cholerae, Shigella flexneri, and Streptococcus pyogenes. As with the other studies and also with the present investigation, the fruit and leaves from the Passiflora family presented antimicrobial activity against the bacteria against which they were tested.

The results presented in this research are consistent with those of other authors, ${ }^{2,5}$ presumably because extracts from the fruit and leaves of the Passiflora family were used, which supports the theory that the fruits from this family are effective as antimicrobial agents against human pathogenic bacteria. However, the results differ with those of another author, ${ }^{2}$ whose findings show a greater antibacterial effect from the same species (Passiflora mollissima) for the preparation of their extract. This may be because although the methodology chosen in the present study is very similar to that of the aforementioned author, it is distinguished by the type of plant.

Interestingly, the cytotoxicity found in the present study indicates that the ethanolic extract of Passiflora mollissima has a highly cytotoxic effect on the fertilized eggs development of sea urchins (Tetrapygus niger) in all its microsolutions at $0.125 \mu \mathrm{L} / \mathrm{mL}$,

$0.25 \mu \mathrm{L} / \mathrm{mL}$ and $0.375 \mu \mathrm{L} / \mathrm{mL}$, with a mean of $100 \%$ lethality at 26 hours after the start of the assay. The cytotoxic activity of the ethanolic Passiflora mollissima extract on the embryonic development of Tetrapygus niger found in this study seems to be consistent with the results observed in the study performed before, in which the authors evaluated the possible cytotoxic activity of the extract of four propolis samples from the state of Minas (eastern Brazil) and two from the state of Paraná (southern Brazil). As also observed in the present study, the methanolic extracts of all the samples were highly active $(97-100 \%)$. The extracts were also prepared with hexane, chloroform, ethyl acetate, and methanol. High activity was documented for all the diluent fractions. ${ }^{34}$

The results seem to be consistent, with a study on the cytotoxic activity of leaves and stems of Passiflora edulis. In this study, petroleum ether and chloroform fractions were used for the ethanolic extract of the leaves and stems of this plant. For this purpose, a brine shrimp (Artemia salina) bioassay was used, in which diluted fractions of the extract were tested against a medium of saline water containing Artemia for 48 hours. After their exposure, a 
24-hour control was performed to observe the lethality rate in Artemia. The results indicated high mortality, which suggests a high cytotoxic activity for the plant. This result is very similar to that of the present study in which, despite the different bioassay (sea urchins), another diluent medium (ethanol) and another plant sample (Passiflora mollissima), the conclusions concur with the possible high cytotoxic effect of the Passiflora family of plants. The results also demonstrate that the plant extract possesses cytotoxic activities. ${ }^{35}$

Although the antibacterial effect of the ethanol extract of the pulp of Passiflora mollissima was evidenced, one way to possibly enhance its antimicrobial effectiveness would be the use of methanol at the time of preparing the extract. Otherwise, another possible method to increase this activity would be to carry out tests of different extracts of several plants together to achieve a potential synergism in the antimicrobial activity. It is not necessary that the ethanol extract be made for each test because the ethanol maintains the chemical properties of the extracts stable.

One of the main limitations of this experimental in vitro research was that it only evaluated the cytotoxic and antibacterial activity of the pulp extract of Passiflora mollissima, so it is recommended to use different fractions from the leaf, seed and peel in future trials; because they could also have a potential antibacterial or antifungal activity. Otherwise, another limitation of this investigation was that only the antimicrobial effect against four bacterial strains was evaluated, therefore it is suggested to demonstrate its effectiveness with other microorganisms of the oral cavity.

However, the results of this study serve as scientific evidence to discover new antimicrobial agents that can be used as potential therapeutic substances, opening in this way a great line of research in relation to this littlestudied natural resource.

\section{CONCLUSION}

In conclusion, an ethanolic extract of the Passiflora mollissima fruit had antimicrobial activity against Streptococcus sanguinis at a $100 \%$ concentration, followed by Streptococcus oralis at the $100 \%$ concentration. It was least effective against the Streptococcus mutans. However, the strain with the lowest antimicrobial activity was Streptococcus mutans, at the $25 \%$ concentration. There was no antifungal effect against the strain of Candida albicans. The minimum inhibitory concentration of ethanolicPassiflora mollissima extract in cultured strains of the microorganisms S. mutans, S. oralis, S. sanguinis was $50 \%(2000 \mu \mathrm{L} / \mathrm{mL})$, and a cytotoxic effect of the ethanolic fruit extract of Passiflora mollissima on the embryonic development of fertilized sea urchin ovules
(Tetrapygus niger) was observed with a mean of $100 \%$ for developmental mortality after 26 hours of exposure.

\section{ACKNOWLEDGMENTS}

Authors wish to thank all the professionals at Universidad Privada San Juan Bautista (UPSJB) and the Universidad Nacional Mayor de San Marcos (UNMSM) who provided the necessary materials to execute all the assays described in the present investigation. This research was supported by the researchers' resources and carried out in the corresponding laboratories of the UPSJB and UNMSM.

\section{REFERENCES}

1. Vila R, Santana A, Pérez R, Valderrama A, Castelli M, Mendonca S, Zacchino S, Gupta M, y Cañigueral S. Composition and biological activity of the essential oil from leaves of Plinia cerrocampanensis, a new source of $\alpha$-bisabolol. BioresourTechnol 2010;101(7):2510-2514.

2. Kannan S, Parimala B, Jayakar B. In Vitro antibacterial activity of various extracts on Leaves of Passiflora mollisima. J Chem Pharm Res 2010; 2(5):225-228.

3. Latha R, Saravana O, Sagaya R, Venkatadri B, Agastian P. Antimicrobial Efficacy and $\alpha$ - Glucosidase Inhibition of Passiflora mollissima Bailey Leaves and IT'S Phytochemical Analisis. J Pharm Health Care Sci 2015;4(4):62-78.

4. Ingale A, Hivrale A. Pharmacological studies of Passiflora sp. and their bioactive compounds. Afr J Plant Sci 2010;4(10): 417-426.

5. Mohanasundari C, Natarajan D, Srinivasan K, Umamaheswari S, y Ramachandran A. Antibacterial Properties of Passiflora foetida L. - a common exotic medicinal plant. Afr J Biotechnol 2007; 6 (23):2650-2653.

6. Razia M, Beulah S, Sivaramakrishnan S. Phytochemical, GC-MS, FT.IR Analysis and Antibacterial Activity of Passiflora Edulis of Kodaikanal Region of Tamilnadu. World J Pharm Sci 2014;3(9):435-441.

7. Shiamala R, Japar B, Muta Z. Assessment of Total Phenolic, Antioxidant, and Antibacterial Activities of Passiflora Species. Scientific World Journal 2014;3(2):1-10.

8. Ghribi L, Nejma A, Besbes M, Harzalla F, Flamini G, Jannet H. Chemical Composition, Cytotoxic and Antibacterial Activities of the Essential Oil from the Tunisian Ononisangustissima L (Fabaceae). J Oleo Sci 2016; 65(4): 339-345.

9. Rios J, Recio M, Villar A. Screening methods for natural products with antimicrobial activity: A review of the literature. J Ethnopharmacol1988; 23(2):127-249.

10. Rosella Camere-Colarossi , Gabriela Ulloa-Urizar, Dyanne Medina-Flores, Stefany Caballero-García, Frank Mayta-Tovalino, Juana del Valle-Mendoza. Antibacterial activity of Myrciaria dubia (Camu camu) against Streptococcus mutans and Streptococcus sanguinis. Asian Pac J Trop Biomed 2016;6(9): 740-744.

11. Bhalodia N, Shukla V. Antibacterial and antifungal activities from leaf extracts of Cassia fistula 1.: An ethnomedicinal plant. J Adv Pharm Technol Res 2011;2(2):104-109.

12. Nascimento G, Locatelli J, Freitas P, Silva G. Antibacterial activity of plant extracts and phytochemicals on antibiotic-resistant bacteria. BrazJ Microbiol 2000;31(4):247-256.

13. Santos F, Bastos E, Uzeda M, Carvalho M, Farias L, Moreira E. Antibacterial activity of Brazilian propolis and fractions 
against oral anaerobic bacteria. J Ethnopharmacol 2002; 80(1):1-7.

14. Chovanová R, Mikulášová M, Vaverková Š. In Vitro Antibacterial and Antibiotic Resistance Modifying Effect of Bioactive Plant Extracts on Methicillin-Resistant Staphylococcus epidermidis. Int J Microbiol 2013; 2(1):1-7.

15. Chaves T, Clementino E, Felismino D, Alves R, Vasconcellos A, Coutinho $\mathrm{H}$ et al. Antibiotic resistance modulation by natural products obtained from Nasutitermescorniger (Motschulsky, 1855) and its nest. Saudi J Biol Sci 2015; 22(4):404-840.

16. Vadhana P, Singh B, Bharadwaj M. Emergence of Herbal Antimicrobial Drug Resistance in Clinical Bacterial Isolates. Pharma Anal Acta. 2015; 6(10):434

17. Patil A, Paikrao H, Patil S. Passiflora Foetida Linn: A Complete Morphological and Phytopharmacological Review. Int J Pharm Bio Sci 2013; 4(1):285-296.

18. Farhana A, Mahmuda H, Laizuman N. Antibacterial, Cytotoxic and Antioxidant Activity of Passiflora Edulis Sims. Eur J Sci Res 2009; 31(4):592-598.

19. Shiamala R, Japar B, Muta Z. Assessment of Total Phenolic, Antioxidant, and Antibacterial Activities of Passiflora Species. Scientific World Journal 2014;3(2):1-10.

20. Birner J, Nicolls J. Passicol, an Antibacterial and Antifungal Agent Produced by Passiflora Plant Species: Preparation and Physicochemical Characteristics. Antimicrob Agents Chemother 1973; 3(1):105-109.

21. Dyanne Medina-Flores, Gabriela Ulloa-Urizar, Rosella CamereColarossi , Stefany Caballero-García , Frank Mayta-Tovalino, Juana del Valle-Mendoza. Antibacterial activity of Bixa orellana L. (achiote) against Streptococcus mutans and Streptococcus sanguinis. Asian Pac J Trop Biomed 2016; 6(5):400-403.

22. Denapaite D, Rieger M, Köndgen S, Brückner R, Ochigava I,Kappeler P, Mätz-Rensing K, Leendertz F, Hakenbeck R. Highly Variable Streptococcus oralis Strains Are Common among Viridans Streptococci Isolated from Primates. mSphere, 2016;1(2): e00041-15.

23. CheonK, Moser S, Wiener H, Whiddon J, Momeni1 S, Ruby J, Cutter G, Childers N. Characteristics of Streptococcus mutans genotypes and dental caries in children, Eur J Oral Sci2013;121(301):148-155.

24. Napimoga N, Höfling J,Klein M, Kamiya R, Gonçalves R. Tansmission, diversity and virulence factors of Sreptococcus mutans genotypes.J Oral Sci 2005;47(2):59-64.
25. Camara L,FernandesV, Meyre S, Guilherme da Cunha M, Tasca A, Ernesto de Carvalho J, Sartoratto A, Garcia V, Mara G, Teixeira M, Ikegaki M, Matias de Alencar M, Rosalen P. Antimicrobial Activity of Essential Oils against Streptococcus mutans and their Antiproliferative Effects. J Evide Based Complementary AlternMed 2012;751435.

26. Corcuera M, Gómez F, Gómez M, Ramos C, Parte M, Alonso $\mathrm{M}$, Prieto J. Qualitative and quantitative agar invasion test based on bacterial colony/biofilm. J Microbiol Methods 2013; 94(3):267-273.

27. Azizi A, Amirzadeh Z, Rezai M, Lawaf S, Rahimi A. Effect of photodynamic therapy with two photosensitizers on Candida albicans. J PhotochemPhotobiol B 2016; 15(8):267-273.

28. Hose J. Potential uses of sea urchin embryos for identifying toxic chemicals: Description of a bioassay incorporating cytologic, cytogenetic andembryologic endpoints. JAppl Toxicol 1985, 5(4):245-254.

29. Fernandes C, Freitas J, Salatino A, Salatino M. Cytotoxic Activity of Six Samples of Brazilian Propolis on Sea Urchin (Lytechinusvariegatus) Eggs. J Evide Based Integr Med 2013:1-4.

30. Figuerola B, ToboadaS, Monleón T, Vázquez J, Ávila C. Cytotoxic Activity of Antarctic Benthic Organisms Against the Common Sea Urchin Sterechinusneumayeri. Oceanography 2013, 1(2):1-9.

31. W. Lin, H. Zhang, G. Beck,"Phylogeny of natural cytotoxicity: Cytotoxic activity of coelomocytes of the purple sea urchin, Arbaciapunctulata," J Exp Zool 2001;290(7);741-750.

32. García-Ruiz A, Girones-Vilaplana A, León P, MorenoD, Stinco C, Meléndez-Martínez A,Ruales J. Banana Passion Fruit (Passiflora mollissima (Kunth) L.H. Bailey): Microencapsulation, Phytochemical Composition and Antioxidant Capacity," Molecules 2017. 22(85):1-12.

33. Costa L, Ferreira M, Lemos T, Pessoa O, Viana G, Cunha G. Toxicity to sea urchin egg development of the quinone fraction obtained from Auxemma oncocalyx. Braz J Med Biol Res 2002; 35(8): 927-930.

34. Gui C, Yuan J, Mo X, Huang H, Zhang S, Gu YC, Ju J. Cytotoxic Anthracycline Metabolites from a Recombinant Streptomyces. J Nat Prod. 2018; 16.

35. Asadujjaman M,Mishuk A, Hossain M. Medicinal potential of Passiflora foetida L. plant extracts: biological and pharmacological activities. JIntegr Med 2014,12(2):121-126. 